\title{
PARÁMETROS GENÉTICOS DE LAS CARACTERÍSTICAS BIOMETRICAS DEL FRUTO Y SEMILLAS EN Pachira aquatica Aubl.
}

\section{GENETIC PARAMETERS OF FRUIT AND SEED BIOMETRIC CHARACTERISTICS IN Pachira aquatica Aubl.}

\author{
Miguel Espitia-Camacho ${ }^{1 *}$, Hermes Araméndiz-Tatis ${ }^{2}$, Carlos Cardona-Ayala ${ }^{3}$ \\ ${ }^{1}$ Ingeniero Agrónomo, PhD., Profesor Titular, Facultad de Ciencias Agrícolas. Universidad de Córdoba, Montería - Colombia. \\ *autor para correspondencia, e-mail: mmespitia@correo.unicordoba.edu.co; ${ }^{2}$ Ingeniero Agrónomo, PhD., Profesor Titular, \\ Facultad de Ciencias Agrícolas. Universidad de Córdoba, Montería - Colombia, e-mail: haramendiz@correo.unicordoba. \\ edu.co; ${ }^{3}$ Ingeniero Agrónomo, PhD., Profesor Titular, Facultad de Ciencias Agrícolas. Universidad de Córdoba, Montería - \\ Colombia, e-mail: cecardona@correo.unicordoba.edu.co
}

\author{
Rev. U.D.C.A Act. \& Div. Cient. 21(1): 33-42, Enero-Junio, 2018 \\ https://doi.org/10.31910/rudca.v21.n1.2018.660
}

RESUMEN

Pachira aquatica es un árbol, cuyos frutos y semillas se aprovechan en la alimentación humana y animal, en regiones tropicales; sin embargo, aún no se han reportado investigaciones de su variabilidad genética para elegir el mejor método de mejoramiento de la especie. El objetivo del estudio fue evaluar los parámetros genéticos de las características biométricas del fruto y sus semillas. El estudio, se realizó en la Universidad de Córdoba, Colombia. Se seleccionaron 10 árboles y de cada uno, 10 frutos al azar. Se llevaron a cabo análisis de varianza y de estimación de parámetros genéticos, para 15 características, de los órganos mencionados. Se detectaron diferencias significativas entre árboles para peso/fruto (PEF), peso de cáscara/fruto (PCF), porcentaje de semillas/fruto (POSF), ancho de semilla (ANS), grosor de semilla (GROS), volumen de una semilla (V1S) y densidad de una semilla (D1S). Los mayores estimadores de variabilidad genética se presentaron en GROS, POSF, PCF y PEF, en donde el componente genético representó más del $50 \%$ de la varianza fenotípica. La heredabilidad más alta, tanto en sentido amplio $\left(h^{2} A>76 \%\right)$ como estrecho $\left(h^{2} E>30 \%\right)$, fue para GROS y POSF. La ganancia genética esperada (GGE), como porcentaje de la media general, fue menor del $10 \%$, en 14 de las 15 características estudiadas, excepto para PCF $(10,86 \%)$, por lo que se espera un progreso genético modesto, para tales características. Se recomienda ampliar la base genética de la población local y hacer ensayos genéticos en varias zonas productoras potenciales.

Palabras clave: Varianza genética, heredabilidad, ganancia genética.
Pachira aquatica is a tree whose fruits and seeds are used for human and animal nutrition in tropical regions. However, no research of its genetic variability have yet been reported to choose the best breeding method for the species. The objective of the study was to evaluate the genetic parameters of the biometric characteristics of the fruit and its seeds. The study was conducted at the Universidad de Córdoba, Colombia. Ten trees were selected and, of each, 10 fruits were randomly selected. Analysis of variance and estimation of genetic parameters were carried out for 15 characteristics of both organs. Significant differences were detected between trees for weight/fruit (FW), weight of husk/fruit (FHW), percentage of seeds/fruit (FSP), seed width (SW), seed thickness (ST), volume of a seed (S1V) and density of a seed (S1D). The greatest estimators of genetic variability were presented in ST, FSP, FHW and FW, where the genetic component represented more than $50 \%$ of the phenotypic variance. The highest heritability, both broad $\left(\mathrm{h}^{2} \mathrm{~A}>76 \%\right)$ and narrow $\left(h^{2} E>30 \%\right)$, was for ST and FSP. The expected genetic gain, as a percentage of the general average, was less than $10 \%$ in 14 of the 15 characteristics studied, except for FHW (10.86\%), therefore a modest genetic progress is expected for such characteristics. It is recommended to increase the local population genetic base and make genetic assays in several potential producing areas.

Key words: Genetic variance, Heritability, genetic gain. 


\section{INTRODUCCIÓN}

Pachira aquatica Aubl. (Malvaceae: Bombacoideae) es un árbol originario de América tropical, que se distribuye desde México hasta el norte de Brasil (Infante-Mata et al. 2011), conocido en Colombia como cacao de monte, predominantemente autoincompatible, cuyos frutos son cápsulas semi-leñosas, dehiscente, de forma general oval, que produce de 18 a 27 semillas de testa fina (Hernández-Montero \& Sosa, 2016; Lobo et al. 2013; Silva et al. 2012).

Posee importancia en la restauración ecológica (HernándezMontero \& Sosa, 2016; Infante-Mata et al. 2014), arborización urbana (Infante-Mata et al. 2011), como planta ornamental y alimentación animal y humana (HernándezMontero \& Sosa, 2016; Li et al. 2009); asimismo, en la medicina, en la producción de cosméticos y en los fungicidas, por las propiedades de las semillas (De Lourdes et al. 2015; Souza et al. 2014; Jorge \& Luzia, 2012). Adicionalmente, proporciona alimento y refugio para la vida silvestre y constituye una barrera natural contra la dispersión de plagas y de enfermedades en cultivos agrícolas (Vale et al. 2014).

En el departamento de Córdoba y en la región Caribe colombiana, $P$. aquatica se encuentra como árboles aislados y es utilizada para el consumo de frutos, de semillas y de madera, en poblaciones vulnerables; no obstante, tiene gran potencial en la conservación de la vida silvestre, restauración y mantenimiento de humedales, suelos y bosques degradados y como barrera natural, para la frontera agrícola (HernándezMontero \& Sosa, 2016; Lobo et al. 2013) y arborización urbana (Infante-Mata et al. 2011), en esta región y el país, en general.

Las estimaciones de parámetros genéticos de las poblaciones en mejoramiento son de gran importancia para el fitomejorador, para conocer la estructura genética de las poblaciones, control genético de las características, su variabilidad genética y orientar la elección del mejor método de mejoramiento, que maximiza las ganancias genéticas por selección (Machado et al. 2016; Hefena et al. 2016; Shafique et al. 2016; Domiciano et al. 2015).

Estudios de parámetros genéticos han sido estimados en varias especies forestales (Maia et al. 2016; Ken-Iti et al. 2016; Dias et al. 2015; Santos et al. 2015), con resultados variables en la magnitud de las estimaciones de los parámetros más importantes, como variabilidad genética, heredabilidad y progreso genético, por selección directa e indirecta. Ellos, han permitido progresos genéticos significativos en la conservación, el uso y la mejora de los caracteres de interés e, igualmente, han coadyuvado al desarrollo y a la obtención de poblaciones superiores, con mayor adaptación, producción y productividad de cultivares, de acuerdo a las necesi- dades, las condiciones y los requerimientos de los productores, transformadores y consumidores finales.

El objetivo del presente trabajo fue estimar los parámetros genéticos para 15 características biométricas del fruto y semillas de Pachira aquatica Aubl., buscando generar información básica para futuros programas de mejoramiento y conservación genética.

\section{MATERIALES Y MÉTODOS}

Localización y época de evaluación. Los frutos y sus semillas fueron procesados y evaluados en el laboratorio de fitomejoramiento de la Universidad de Córdoba, entre enero y junio de 2016, de árboles aislados de $P$. aquatica, de origen genético desconocido, cosechados al azar, ubicados en el área urbana y periurbana de Montería, Colombia, perteneciente a la zona media del bosque seco tropical del valle del Sinú (Palencia et al. 2006).

Material genético. Se evaluaron 10 árboles de $P$. aquatica muestreados aleatoriamente, de los cuales, se tomaron al azar 10 frutos de libre polinización de cada uno, para un total de 100 frutos. Los árboles muestreados fueron marcados y georreferenciados, para continuar futuros estudios. Se muestrearon únicamente 10 árboles, porque en la población estudiada solo se encontró esta cantidad, con 10 o más frutos totalmente desarrollados, completos y maduros. Dado que la especie es alógama (Hernández-Montero \& Sosa, 2016), las semillas de cada árbol se consideraron como una familia de hermanos medios. Al momento de la colecta de los frutos, los árboles presentaron de 5 a $15 \mathrm{~m}$ de altura, 10 a 25 años de edad y 15 a $30 \mathrm{~cm}$ de diámetro a la altura del pecho (DAP), aproximadamente (Figura 1).

Variables de respuesta. Se estimaron parámetros de 15 variables de respuesta relacionadas con las características biométricas del fruto y semilla de $P$. aquatica, a saber: peso fresco/fruto (PEF), largo/fruto (LAF), grosor de la parte media del fruto (GROF), peso de cáscara/fruto $(\mathrm{PCF})$, número de semillas/fruto (NSF), peso de semillas/fruto (PSF), porcentaje de semillas/fruto (POSF), largo de semilla (LAS), ancho de semilla (ANS), grosor de semilla (GROS), peso de una semilla (P1S), volumen de una semilla (V1S), densidad de una semilla (D1S), peso de 100 semillas (P100S) y número de semillas/kilogramo (NSKG).

El PEF (g), LAF (cm), PCF (g), NSF (\#), PSF (g), POSF = (PSF/PEF)*100 (\%) fueron estimados de forma individual, para cada fruto dentro de cada árbol; el P1S $(\mathrm{g})$, LAS $(\mathrm{cm})$, ANS $(\mathrm{cm})$ y el GROS $(\mathrm{cm})$ fueron estimados como el promedio de las estimaciones individuales respectivas, de la semilla presente dentro de cada fruto (PSF/NSF), en cada árbol; el V1S fue estimado en $\mathrm{ml}$, como el incremento promedio en 

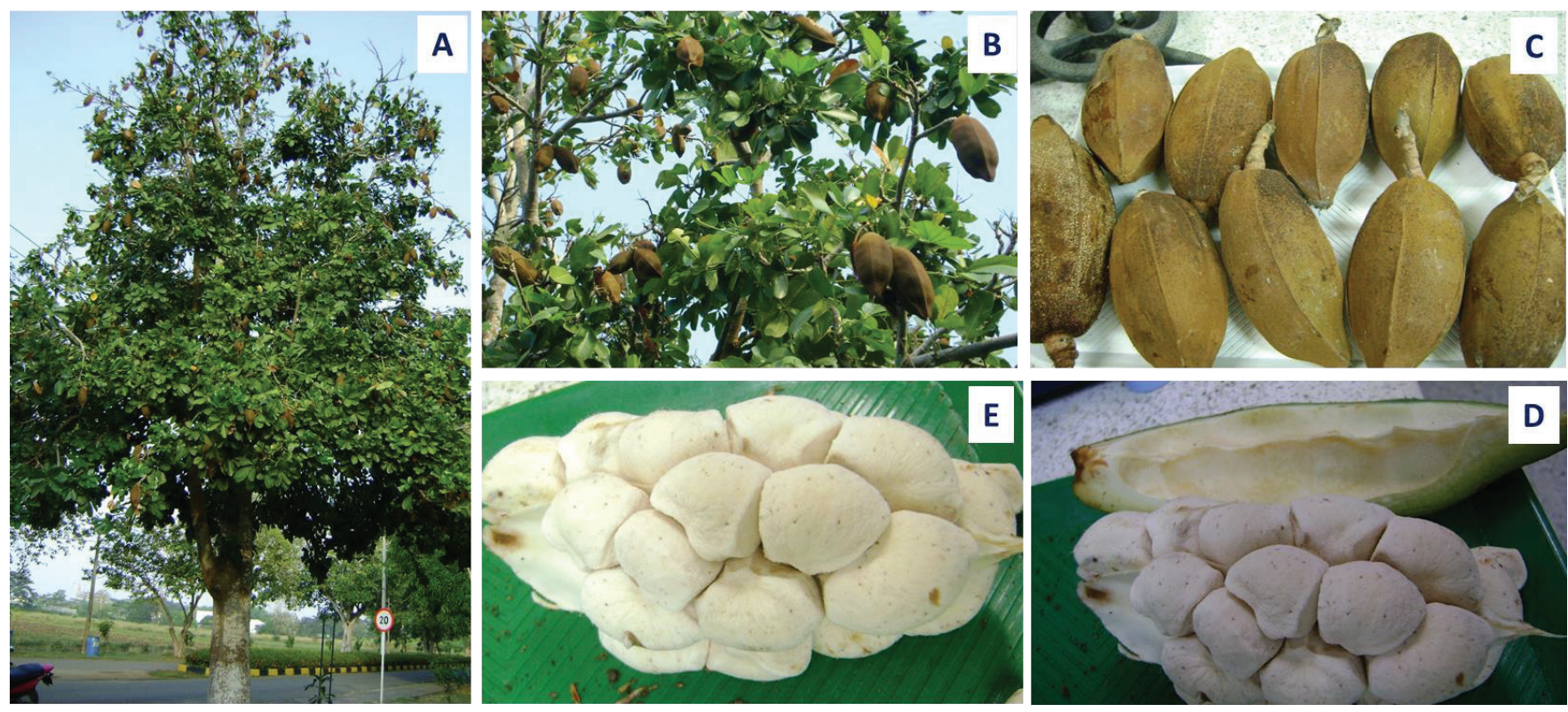

Figura 1. A) Árbol aislado; B) Ramas con frutos; C) Frutos cosechados; D) y E) Semillas agrupadas recién extraídas del fruto.

volumen que se genera en una probeta, con volumen de agua destilada conocido, cuando se introdujo una muestra de cinco semillas tomadas al azar, de cada fruto, dentro de cada árbol y la D1S fue estimada a partir de la relación (P1S/V1S).

Análisis de datos. Las 10 familias de hermanos medios, se evaluaron mediante análisis de varianza a una vía, para estimar la variación entre y dentro de familias o poblaciones. Además, se estimaron los parámetros genéticos: coeficientes de variación fenotípicos (CVF), varianza fenotípica media entre familias de hermanos medios (VF), varianza genética media entre familias de hermanos medios (VG), varianza ambiental media entre familias de hermanos medios
(VA), varianza genética aditiva media entre familias de hermanos medios (VGA), coeficientes de variación genotípicos (CVG), heredabilidad en sentido amplio $\left(\mathrm{h}^{2} \mathrm{~A}\right)$, heredabilidad en sentido estricto $\left(\mathrm{h}^{2} \mathrm{E}\right)$, relación entre $(\mathrm{CVG} / \mathrm{CVe})$ y estadísticas descriptivas para las 15 variables de respuesta consideradas. Los análisis estadísticos y parámetros genéticos, se realizaron mediante el uso del programa computacional de libre acceso GENES, versión Windows GENES V.2014.6.1 (Cruz, 2016).

Los parámetros genéticos fueron estimados con el programa GENES, a partir de los resultados del análisis de varianzas y teniendo en cuenta los cuadrados medios esperados (Tabla 1).

Tabla 1. Fuentes de variación (FV), grados de libertad (GL) y cuadrados medios (CM) esperados para el análisis de varianza, bajo el uso del diseño completamente al azar con 10 tratamientos y 10 repeticiones.

\begin{tabular}{|l|c|c|l|}
\hline \multirow{2}{*}{ FV } & \multirow{2}{*}{ GL } & CM & \multicolumn{1}{c|}{ CM Esperados } \\
\cline { 3 - 4 } & & & \\
\hline Árboles & $(\mathrm{a}-1)=9$ & $\mathrm{CM}$ & $\sigma^{2}+r \sigma^{2} \mathrm{HM}$ \\
\hline Error & $\mathrm{r}(\mathrm{a}-1)=90$ & $\mathrm{CME}$ & $\sigma^{2}$ \\
\hline Total & $(\mathrm{ra}-1)=99$ & & \\
\hline
\end{tabular}

a: número de árboles (10); r: repeticiones (10); СМнм: Cuadrados medios para familias de hermanos medios (HM); CMЕ: Cuadrados medio del error o residual; $\sigma_{\mathrm{HM}}{ }=$ varianza media entre familias de $\mathrm{HM} ; \sigma_{\mathrm{HM}}{ }^{2} \sigma^{2}{ }_{\mathrm{G}}=$ varianza genética $=\left(\mathrm{CM} M_{\mathrm{HM}}-\right.$ $\mathrm{CM} / \mathrm{E} / \mathrm{r} ; \sigma^{2}=$ varianza ambiental de observaciones individuales $=\mathrm{CM}_{\mathrm{E}} ; \sigma_{\mathrm{E}}^{2}=$ varianza ambiental de media entre familias $\mathrm{HM}$

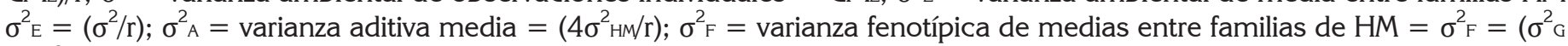
$+\sigma^{2}$ E). (Hallauer \& Miranda, 1981). 
La heredabilidad en sentido amplio $\left(\mathrm{h}^{2} \mathrm{~A}\right)$ y estrecho $\left(\mathrm{h}^{2} \mathrm{E}\right)$, para cada variable, fue estimada de la forma clásica, como se describe a continuación:

$\mathrm{h}^{2} \mathrm{~A}=\left(\sigma^{2} \mathrm{G} / \sigma_{\mathrm{F}}^{2}\right)^{*} 100$

$h^{2}=\left(\sigma^{2} \mathrm{~A} / \sigma^{2}\right)^{*} 100$

Donde, $\sigma^{2}$ : varianza genética media para familias de hermanos medios; $(\mathrm{HM}) ; \sigma_{\mathrm{F}}^{2}$ : Varianza fenotípica media para familias de $H M ; \sigma^{2}$ : Varianza genética aditiva media para familias de HM.

La ganancia genética esperada (GGE), para cada variable, se estimó, a través de la siguiente ecuación (Hallauer \& Miranda, 1981):

$\mathrm{GGE}=\mathrm{k}^{*} \sigma * \mathrm{~h}^{2} \mathrm{E}$

Donde $\mathrm{k}={ }^{\mathrm{F}}$ Diferencial de selección al $5 \%$ de intensidad de selección, equivalente a 2,06; $\sigma_{F}=$ Desviación estándar fenotípica; $h^{2}=$ Heredabilidad en ${ }^{\mathrm{F}}$ sentido estrecho.

La GGE, también se expresó en porcentaje:

GGE $(\%)=($ GGE $/$ Media $) * 100$
El rango de la GGE (\%) fue clasificado, como: bajo $(<10 \%)$, moderado (10-20\%) y alto (>20\%).

\section{RESULTADOS Y DISCUSIÓN}

Características biométricas de frutos y semillas. Los cuadrados medios del análisis de varianza, detectaron diferencias estadísticas significativas $(\operatorname{Pr}<0,05$ y $\operatorname{Pr}<0,01)$, para siete de las 15 variables dependientes consideradas: PEF, PCF, POSF, ANS, GROS, V1S y D1S (Tabla 2); esto evidencia, la posible existencia de variabilidad genética entre los árboles en estudio y aumenta la probabilidad de lograr progresos genéticos sucesivos en el tiempo, por la selección o clonación de árboles, con características de frutos y de semillas deseables, en tales caracteres (Silva et al. 2012; De Oliveira et al. 2007), lo que concuerda con lo reportado por Cardona-Medina \& Muriel (2015); Salla et al. (2015) y Marcal et al. (2015), en Escobedia grandiflora, Plinia cauliflora y Euterpe edulis, respectivamente.

Tabla 2. Cuadrados medios y nivel de significancia estadística del análisis de varianza para 15 características biométricas de frutos y semillas de Pachira aquatica, en Montería, Colombia.

\begin{tabular}{|c|c|c|}
\hline \multirow{2}{*}{ Variables } & \multicolumn{2}{|c|}{ CM de FV } \\
\hline & Árboles $(\mathrm{GL}=9)$ & Error $(G L=90)$ \\
\hline $\operatorname{PEF}(g)$ & $46664,61^{*}$ & 19680,78 \\
\hline $\operatorname{LAF}(\mathrm{cm})$ & 5,6460ns & 3,6456 \\
\hline GROF $(\mathrm{cm})$ & $0,7233 n s$ & 0,6483 \\
\hline $\mathrm{PCF}(\mathrm{g})$ & $31043,22^{* * *}$ & 10443,78 \\
\hline NSF (\#) & $76,08 \mathrm{~ns}$ & 54,84 \\
\hline PSF (g) & $2580,72 n s$ & 1785,23 \\
\hline POSF (\%) & $24,72 * *$ & 5,85 \\
\hline LAS $(\mathrm{cm})$ & $0,0963 n s$ & 0,0506 \\
\hline ANS $(\mathrm{cm})$ & $0,0849 *$ & 0,0400 \\
\hline GROS $(\mathrm{cm})$ & $0,2776 * *$ & 0,0491 \\
\hline P1S (g) & $1,00 \mathrm{~ns}$ & 0,68 \\
\hline V1S (ml) & $1,867^{*}$ & 0,899 \\
\hline D1S (g/ml) & $0,0361^{*}$ & 0,0143 \\
\hline P100S (g) & $10002,03 n s$ & 6801,98 \\
\hline NSKG (\#) & $2004,05 \mathrm{~ns}$ & 1332,54 \\
\hline
\end{tabular}

CM: cuadrados medios; FV: fuentes de variación; GL: grados de libertad; *, **: significativos al $5 \%$ y $1 \%$ de probabilidad, respectivamente; ns: no significativos; PEF: Peso/fruto; LAF: Largo/fruto; GROF: grosor/fruto; PCF: peso de la cáscara/fruto; NSF: número de semillas/fruto; PSF: peso de semilla/fruto; POSF: porcentaje de la semillas/fruto; LAS: largo de semilla; ANS: ancho de semilla; GROS: grosor de semilla; P1S: peso de una semilla; V1S: volumen de una semilla; D1S: densidad de una semilla; P100S: peso de 100 semillas; NSKG: número de semillas/kilogramo. 
Las diferencias detectadas para las características biométricas de frutos y de semillas entre los árboles, se pueden explicar por el efecto diferencial de la constitución genética, a nivel de individuos, tipo de población y polinización cruzada, condiciones edafoclimáticas, edad de la planta madre y nivel de madurez a cosecha, los cuales, afectan las características biométricas y fisiológicas del fruto y las semillas (Silva et al. 2015; Ferreira \& Barbosa, 2015; Rodrigues et al. 2015).

La ausencia de diferencias significativas en las otras características de los frutos y las semillas restantes permiten deducir alta homogeneidad fenotípica y homocigosidad genotípica, en los 10 árboles estudiados, posiblemente, por un efecto fundador u origen genético común estrecho y con alto parentesco genético, entre ellos, a raíz de polinización cruzada entre pocos individuos emparentados genéticamente, preexistente en la región, que pudieron dar origen a la semillas de donde se derivaron los árboles muestreados, en la zona de estudio, quienes, a su vez, por ende, exhiben un comportamiento muy similar, para tales variables y, por ello, reducen la posibilidad de lograr avance genético importante, mediante la selección de árboles en estos caracteres (Machado et al. 2016; Shafique et al. 2016; Rodrigues et al. 2015; Silva et al. 2015).

Parámetros genéticos. Los parámetros genéticos estimados para las características biométricas del fruto y las semillas de $P$. aquatica, se presentan en la tabla 3. Los parámetros genéticos relacionados con variabilidad y heredabilidad fueron diferentes en magnitud para las 15 variables consideradas, como se esperaba. Los CVF oscilaron entre $8,66 \%$, para POSF y $21,26 \%$, en el PSF. Estos valores están estrechamente relacionados con los niveles de significancia estadísticas detectadas en el análisis de varianza (Tabla 2). Además, una buena precisión experimental, como lo sostienen Ken-Iti et al. (2016), por acusar $\mathrm{CV}<20 \%$, con excepción de PSF (Tabla 2); ello permite inferir alta confiabilidad de los valores fenotípicos en representar los valores genotípicos, sobre todo, cuando se trabaja con poblaciones naturales, sometidas a altos efectos variables de los factores bióticos y abióticos ambientales, propios del Caribe húmedo colombiano.

Las estimaciones de las varianzas genéticas medias entre familias de hermanos medios (VG), como componentes de las varianzas fenotípicas medias (VF), resultaron ser superiores a las varianzas ambientales medias (VA), en las variables: GROS, POSF, PCF, D1S, PEF, ANS y V1S, ya que representan más del $50 \%$ de la VF, lo que indica, la existencia de variabilidad genética aditiva y no aditiva en la población estudiada, para estos caracteres biométricos de frutos y sus semillas. En tales variables, la variación fenotípica detectada estuvo explicada por más del $50 \%$, por variaciones, debido a efectos ambientales. Esta situación, se corrobora con las magnitudes obtenidas en VGA, CVG y los índices de variabilidad genética $\mathrm{b}$, en donde se puede observar una tendencia similar a la descrita para los componentes de varianzas genéticas en las mismas variables; situaciones similares han sido reportadas en otros estudios (Hefena et al. 2016; Ken-Iti et al. 2016; Santos et al. 2015).

Las diferencias en las magnitudes estimadas entre los componentes de varianza genética media (VG) y varianza genética aditiva (VGA), se sustentan, porque la VG es originada por genes que actúan en forma aditiva y no aditiva, en tanto que VGA es sustentada por los genes que actúan en forma aditiva. Este tipo de variación genética aditiva es considerado de gran interés en el mejoramiento genético de poblaciones, ya que es el que responde a la selección de individuos superiores, permite aumentar la frecuencia de alelos favorables en los caracteres de interés en la siguiente generación y, por ende, coadyuva a que se logre progreso genético importante por selección en las poblaciones en generaciones siguientes, sobre todo en especies alógamas, como $P$. aquatica, en las variables GROS y PEF, que fueron los caracteres que exhibieron los mayores valores en CVG $(8,88 \%$ y $7,28 \%)$ y b $(0,68$ y 0,37 ) (Tabla 3); sin embargo, hay que tener en cuenta que la variabilidad observada para un determinado carácter no siempre es transmitida de generación a generación, evidenciando que el efecto del ambiente puede enmascarar la variación genética aprovechable, por ello, la selección es más efectiva cuando actúa sobre caracteres de alta heredabilidad y que tienen asociación con la producción u otro carácter de importancia económica (Hefena et al. 2016; Ken-Iti et al. 2016; Moraes et al. 2014; Silva et al. 2013).

Las mayores estimaciones de heredabilidad $\left(h^{2} A\right)$ y $\left(h^{2} E\right)$, se presentaron en GROS, con valores de $82,32 \%\left(h^{2} A\right)$ y $32,93 \%$ $\left(\mathrm{h}^{2} \mathrm{E}\right)$, respectivamente, sobresaliendo como los caracteres de mayor probabilidad para lograr avances por selección, ya que exhiben buena variabilidad genética heredable. A su vez, los menores valores de $\mathrm{h}^{2} \mathrm{~A} \mathrm{y} \mathrm{h}^{2} \mathrm{E}$, se obtuvieron en GROF, con magnitudes de 10,37 y 4,29\%, en su orden (Tabla 3). Esto constituye un limitante para el progreso de la selección y es factible que el efecto fundador esté incidiendo en ello, dado el número de individuos evaluados, lo cual, a futuro, amerita la introducción de germoplasma, para generar nuevas recombinaciones genéticas y, con ello, mayor variabilidad. Los mayores valores obtenidos a favor de la $\mathrm{h}^{2} \mathrm{~A}$, con relación a los obtenidos en $h^{2}$, en cada una de las variables evaluadas, se expresan, porque la $h^{2} A$ mide y explica el porcentaje de la VF, que se debe a la VG total, en tanto que la $h^{2} E$ mide la proporción de la VF, que solo es explicada por la VGA, la cual, es de naturaleza heredable (Solangi et al. 2016; Maia et al. 2016; Ken-Iti et al. 2016). 


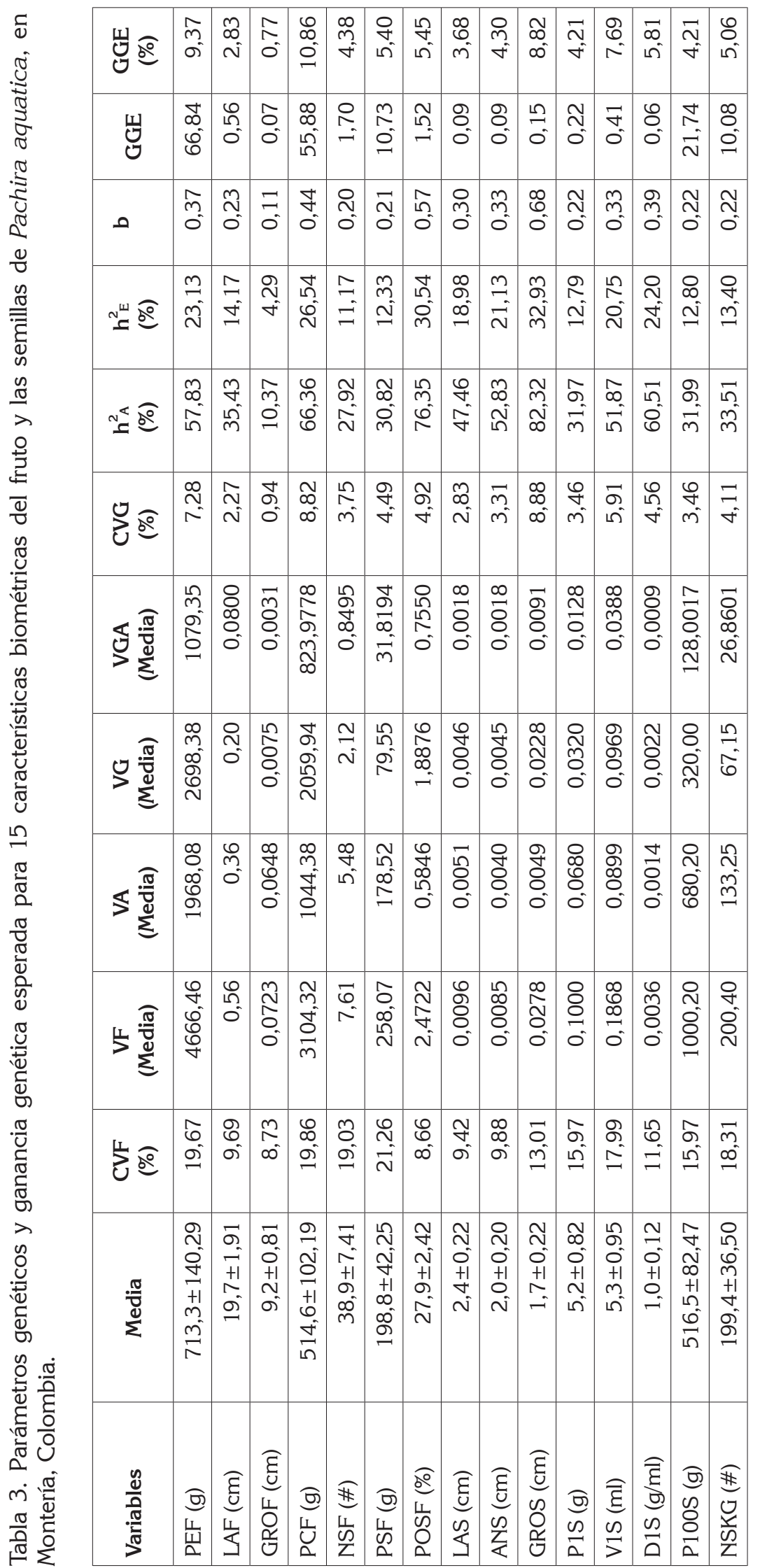

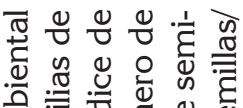
है

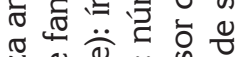

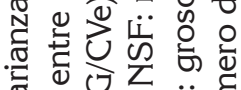

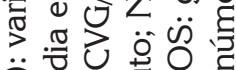

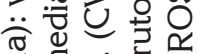

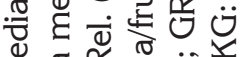

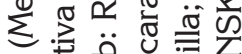

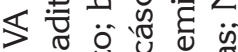
을

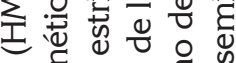

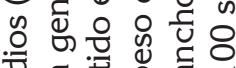
ช สู छ 它

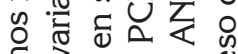
ธิ

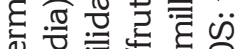
舟 \& 我它

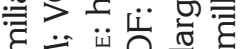

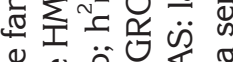

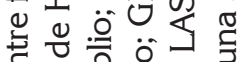

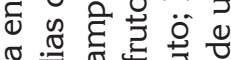
. 뜬

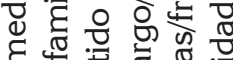
हथ

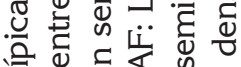

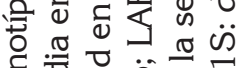

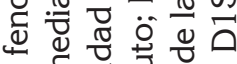
중 तี ชु 通过过

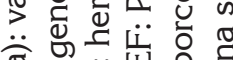
高宁㟧

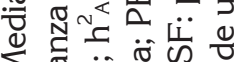
$\sum$.

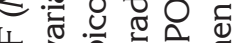

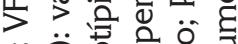

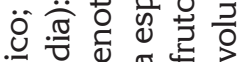

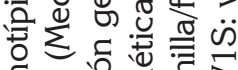

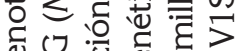

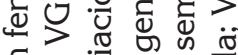
ป

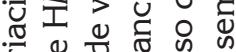

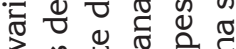
व

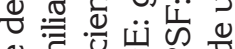
ह छ ट्य

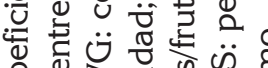
ठำ 응

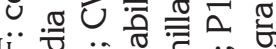

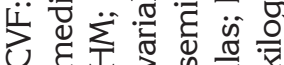


Las magnitudes y las clasificaciones de $\mathrm{h}^{2}$ A obtenidas para las 15 variables, se agruparon con heredabilidad alta (>50\%): GROS, POSF, PCF, D1S, PEF, ANS y V1S; heredabilidad media (20 - 50\%): LAS, LAF, NSKG, P100S, P1S, PSF y NSF y heredabilidad baja (<20\%): GROF. A su vez, según los valores de $h^{2} \mathrm{E}$, las variables se clasificaron en heredabilidad media (20 - 50\%): GROS, POSF, PCF, D1S, PEF, ANS y V1S y baja (<20\%): LAS, LAF, NSKG, P100S, P1S, PSF, NSF y GROF (Tabla 3). En general, se considera que la heredabilidad en sentido estrecho $\left(\mathrm{h}^{2} \mathrm{E}\right)$ es la más importante en el mejoramiento por selección, dado que determina cuánto de la apariencia fenotípica de las plantas es el reflejo exacto de su valor genético. Estimaciones altas de heredabilidad asociadas con mayor progreso genético garantizan y confirman la respuesta a la selección en el desarrollo de nuevos genotipos, con características deseables (Solangi et al. 2016; Maia et al. 2016; Ken-Iti et al. 2016).

Los parámetros genéticos VG, VGA, CVG e índice de variabilidad b explican y favorecen la alta heredabilidad detectada en sentido amplio $\left(h^{2} A\right)$ y estrecho $\left(h^{2} E\right)$, para GROS, POSF, PCF, D1S, PEF, ANS y V1S, resultados similares han sido reportados en diferentes variables de interés en Platonia insignis (Maia et al. 2016) y Euterpe oleracea (Ken-Iti et al. 2016).

Las estimaciones de los parámetros genéticos de los caracteres PEF, GROS y POSF indican que son los más importantes para el mejoramiento genético de esta especie, de forma que, la selección de frutos pesados con semillas grandes y un alto porcentaje de semillas por fruto, permitiría la obtención de plántulas vigorosas, tanto para la restauración de ambientes como para la alimentación humana y animal. Estos caracteres resultan de interés económico para las plantaciones, la comercialización, la industrialización y el consumo (Jorge \& Luzia, 2012; Hernández-Montero \& Sosa, 2016). Igualmente, su conocimiento constituye la base fundamental para el éxito de futuros programas de conservación de recursos genéticos y mejoramiento poblacional de la especie, en razón a que, de ellos, depende el mantenimiento de la variabilidad genética, selección de individuos superiores y progreso genético (Paramesh et al. 2014; Farias-Neto et al. 2013).

La ganancia genética esperada, expresada como porcentaje (GGE \%) de la media general de las 10 familias de hermanos medios, fue menor del $10 \%$, para 14 de las 15 variables relacionadas con los aspectos biométricos de frutos y semillas, clasificándose el avance genético esperado como bajo. La excepción a la anterior situación es dada por la variable peso de cáscara/fruto (PCF), que muestra una GGE media, con valor de 10,86\%; no obstante, aunque esta característica juega un papel importante en la protección y en la conservación del fruto y sus semillas, no es atractivo su incremento como un componente deseable para aumentar la producción de frutos/árbol, semillas/fruto o la calidad de las semillas, para mejorar los ingresos al productor de P. aquatica (HernándezMontero \& Sosa, 2016; Silva et al. 2012; Jorge \& Luzia, 2012). A pesar de lo anterior, las mejores GGE, se obtendrían en el PEF y GROS, con incrementos del 9,37 y 8,82\%, respectivamente, con respecto a la media general. Este avance genético representa incrementos esperados de $66,84 \mathrm{~g}$, para PEF y $0,15 \mathrm{~cm}$, en GROS (Tabla 3), lo que indica que la selección de plantas con frutos de buen crecimiento y desarrollo, es posible como resultado de una buena fecundación y así obtener semillas de buena calidad fisiológica, que dada su capacidad de dispersión, de crecimiento rápido y la gran reserva de almidón de los cotiledones, la hacen una especie competitiva en zonas de baja o nula salinidad, en humedales arbóreos tropicales (Infante-Mata et al. 2014).

La baja GGE esperada encontrada en este estudio puede estar explicada, según varios autores (Machado et al. 2016; Shafique et al. 2016; Hernández-Montero \& Sosa, 2016), por la reducida variabilidad genética detectada en el germoplasma evaluado, a raíz del efecto fundador, lo cual, sugiere una base genética estrecha y origen genético común de la población de $P$. aquatica, en Montería, que se expresa en alto nivel de homogeneidad fenotípica y homocigosidad genotípica por endogamia.

La GGE obtenida para GROS y PEF, en la siguiente generación, puede ser mayor cuando se clonan los árboles superiores seleccionados, que cuando se usa su semilla sexual de polinización abierta, porque en la clonación, se captura el $100 \%$ de la información genética (efectos genéticos aditivos y no aditivos) de los árboles seleccionados, mientras que cuando se utiliza semilla sexual originada de libre polinización, solo se captura el 50\% de la información genética de los árboles madre superiores, por ser una especie alógama (Ken-lti et al. 2016; Maia et al. 2016; Farias-Neto et al. 2013).

Una prueba de progenie o clonal de los árboles seleccionados, replicada en al menos tres ambientes diferentes, es aceptable y deseable en este tipo de programas de mejoramiento, ya que permitiría, después del raleo genético, contar con sendos huertos semilleros de primera generación, que ofrecerían semilla de excelente calidad genética, para nuevas siembras comerciales. De igual forma, se contaría con material vegetal para clonar los mejores árboles de las mejores familias en las poblaciones de los huertos, con adaptación específica en cada ambiente. Esta estrategia haría posible que los árboles definitivos de $P$. aquatica se crucen entre sí, forzando su recombinación genética, la acumulación de alelos favorables en la población y, por consiguiente, la producción de semilla de calidad genética superior, para continuar con el proceso de mejoramiento (Farias-Neto et al. 2013; Murillo et al. 2012; Batista et al. 2012). 
Como es de esperarse, es posible que se encuentren genotipos deseables en otros municipios, departamentos, instituciones, empresas, organizaciones o países, por lo tanto, el grupo de árboles que se seleccionen para dar inicio a un programa de mejoramiento genético de $P$. aquatica, en Córdoba y la región Caribe, como lo señalan Murillo et al. (2012), Farias-Neto et al. (2013) y Ken-Iti et al. (2016), permitirá alianzas e intercambio de material genético con otras empresas u organizaciones, con bancos de germoplasma o programas de mejoramiento genético de esta especie. Esta estrategia, junto con la introducción de procedencias, hará posible reducir costos, aumentar variabilidad genética y reducir tasas de endogamia en la población de mejoramiento, lo que permitiría seguir obteniendo ganancias genéticas, durante varias generaciones (Murillo et al. 2012).

Los resultados obtenidos sugieren un progreso genético moderado en el mejoramiento de $P$. aquatica, en Córdoba. De acuerdo con Batista et al. (2012) y Maia et al. (2016), este avance, integrado con el proceso de clonación de los mejores árboles élites, puede constituir el complemento ideal de un programa de mejoramiento genético, para esta especie. Adicionalmente, hacen prever un aporte importante a la productividad, la competitividad y la sostenibilidad de la producción de frutos y semillas de $P$. aquatica, en esta zona del Caribe colombiano, de acuerdo con las exigencias de calidad del mercado local, nacional e internacional, al cual, se pretende llegar; no obstante, es necesario corroborar este progreso genético, mediante el establecimiento de ensayos genéticos en diferentes ambientes de la región Caribe.

El progreso genético moderado en el mejoramiento de las características biométricas de frutos y semillas de $P$. aquati$c a$, en el área de influencia de Montería, permite considerar la introducción de recursos genéticos, para aumentar variabilidad genética y reducir tasas de consanguinidad en la población de estudio.

Las mayores estimaciones de heredabilidad en sentido amplio $\left(h^{2} \mathrm{~A}\right)$ y estrecho $\left(\mathrm{h}^{2} \mathrm{E}\right)$, se presentaron en GROS y POSF, con valores mayores de $76 \%\left(h^{2} A\right)$ y $30 \%\left(h^{2}\right)$.

La ganancia genética esperada, expresada como porcentaje (GGE \%) de la media general, fue menor del $10 \%$, para 14 de las 15 las variables estudiadas, clasificándose como baja, excepto para la variable PCF, que mostró una GGE media, con valor de $10,86 \%$.

Para incrementar la ganancia genética en el mejoramiento de esta especie, se sugiere tener en cuenta el grosor de la semilla (GROS) y porcentaje de la semilla/fruto (POSF), por sus valores de heredabilidad en sentido amplio $\left(h^{2} \mathrm{~A}>76 \%\right)$ y estrecho $\left(h^{2} \mathrm{E} .>30 \%\right)$, bajo las condiciones ambientales de estudio.
Es necesario corroborar la GGE obtenida, mediante ensayos genéticos en varias zonas productoras de Montería, de Córdoba y de Colombia.

Conflicto de intereses: El manuscrito fue preparado y revisado con la participación de todos los autores, quienes declaramos que no existe conflicto de intereses que ponga en riesgo la validez de los resultados presentados.

\section{BIBLIOGRAFÍA}

1. BATISTA, C.M.; MENEZES F., M.L.; DE MORAES, M.A.; ZANATTO SCATENA, A.C.Z; DOS SANTOS, P.C.; ZANATA, M., TEIXEIRA DE MORAES, M.L. SEBBENN, A.M. 2012. Estimativas de parâmetros genéticos e a variabilidade em procedências e progênies de Handroanthus vellosoi. Pesq. Flor. Bras. 32(71):269-276.

2. CARDONA-MEDINA, E.; MURIEL, S.B. 2015. Seed germination and plant development in Escobedia grandiflora (Orobanchae): Evidence of obligate hemiparasitism? Acta Biol. Colomb. 20(3):133-140.

3. CRUZ, C.D. 2016. Programa Genes V.2014.6.1 Aplicativo computacional em genética e estatística. Disponible desde Internet en http://www,ufv,br/dbg/ genes/genes,htm [con acceso el 22/09/2016].

4. DE LOURDES, B.D.A.; DE AZEVEDO, C.C.; AZEVEDO, F.D.L.A. 2015. Propriedades funcionais das proteínas de amêndoas da munguba (Pachira aquatica Aubl.). Rev. Bras. Frutic. 37(1):193-200.

5. DE OLIVEIRA, L.Z.; CESARINO, F.; MORO, F.V.; PANTOJA, T.D.F.; SILVA, B.M.D.S. 2007. Morfologia do fruto, da semente, germinação e plântula de Pachira aquatica Aubl. (Bombacaceae). Revista Brasileira de Biociências. 5(1):840-842.

6. DIAS, P.C.; XAVIER, A.; DE RESENDE, M.D.V.; BIERNASKI, F.A.; ESTOPA, R.A. 2015. Genetic parameters for propagation capacity of Pinus taeda by somatic embryogenesis. Rev. Arvore. (Brasil). 39(6):1093-1102.

7. DOMICIANO, G.P.; ALVES, A.A.; LAVIOLA, B.G.; DE CONCEIÇÃO, L.D.H.C. 2015. Genetic parameters and diversity in progenies from Macaw Palm based on morphological and physiological traits. Ciência Rural. 45(9):1599-1605.

8. FARIAS-NETO, J.T.; CLEMENT, CH.R.; RESENDE, M.D.V. 2013. Estimates of genetic parameters and 
selection gain for fruit production in open-pollinated progenies of peach palm in the State of Pará, Brazil. Bragantia. (Brasil). 72(2):122-126.

9. FERREIRA, R.A.; BARBOSA, S.S.B. 2015. Caracterização morfológica de frutos, sementes, plântulas e mudas de pau-brasil (Caesalpinia echinata Lamarck). Rev. Árvore. 39(3):505-512.

10. HALLAUER, A.R.; MIRANDA, F.J.B. 1981. Quantitative Genetics in Maize Breeding. Second Edition. Iowa State Univ. Press. Iowa (USA). 468p.

11. HEFENA, A.G.; SULTAN, M.S.; ABDEL-MONEAM, M.A.; HAMMOUD, S.A.; BARUTÇULAR, C.; EL-SABAGH, A. 2016. Assessment of Genetic Variability and Correlation Coefficient to Improve Some Agronomic Traits in Rice. Journal of Experimental Agriculture International. (USA).14(5):1-8.

12. HERNÁNDEZ-MONTERO, J.R.; SOSA, V.J. 2016. Reproductive biology of Pachira aquatica Aubl. (Malvaceae: Bombacoideae): a tropical tree pollinated by bats, sphingid moths and honey bees. Plant Species Biology. (USA). 31(2):125-134.

13. INFANTE-MATA, D.; PATRICIA MORENO-CASASOLA, P.; CAROLINA MADERO-VEGA, C. 2014. ¿Pachira aquatica, un indicador del límite del manglar? Rev. Mex. Biodiversidad. 85(1): 143-160.

14. INFANTE-MATA, D.; MORENO-CASASOLA, P.; MADERO-VEGA, C.; CASTILLO-CAMPOS， G.; WARNER, B.G. 2011. Floristic composition and soil characteristics of tropical freshwater forested wetlands of Veracruz on the coastal plain of the Gulf of Mexico. Forest Ecology and Management. (USA). 262(1):1514-1531.

15. JORGE, N.; LUZIA, D.M.M. 2012. Caracterização do óleo das sementes de Pachira aquatica Aublet para aproveitamento alimentar. Acta Amazônica. (Brasil). 42(1):149-156.

16. KEN-ITI, Y.G.; DOS SANTOS, R.G.; MOCHIUTTI, S.; PEREIRA, B.A.; DE QUEIROZ, L.J.A.; GOMES, F.R.; BEZERRA, C.I. 2016. Estimativas de parâmetros genéticos para caracteres de frutos em açaizeiros no Amapá. Ciência Florestal. (Brasil). 26(3):985-993.

17. LI, Q.; DENG, M.; CHEN, J.; HENNY, R.J. 2009. Effects of light intensity and paclobutrazol on growth and interior performance of Pachira aquatica Aubl. Hortscience. (USA). 44(5):1291-1295.
18. LOBO, J.; SOLÍS, S.; FUCHS, E.J.; QUESADA, M. 2013. Individual and temporal variation in outcrossing rates and pollen flow patterns in Ceiba pentandra (Malvaceae: Bombacoidea). Biotropica. (USA) 45(1):185-194.

19. MACHADO, E.L.; SILVA, S.A.; FERNANDES, L.D.S.; BRASILEIRO, H.S. 2016. Variabilidade genética e homozigose em uma população F4 de mamoneira por meio de marcadores microssatélites. Bragantia. (Brasil). 75(3):307-313.

20. MAIA, M.C.C.; MACEDO, L.M.; LOPES, V.L.F.; ALVES, A.J.P.; OLIVEIRA, L.C.; RESENDE, M.D.V. 2016. Estimates of genetic parameters using RELM/BLUP for intrapopulational genetic breeding of Platonia insignis Mart. Rev. Árvore. (Brasil). 40(3):561-573.

21. MARCAL, T.S.; FERREIRA, A.; DOS SANTOS, W.B.O.; SOLER, J.H.G.; DASILVA, M.F.F. 2015. Correlações genéticas e análise de trilha para caracteres de fruto da palmeira juçara. Rev. Bras. Frutic. 37(3):692-698.

22. MORAES, C.B. DE; FERNANDES, B.T.; TEIXEIRA, L.G.; ZIMBACK, L.; VAGNER, T.E.; CHAVES, R.; TEIXEIRA, M.L. DE M.; SEIZO, M.E. 2014. Estimativas dos parâmetros genéticos para seleção de árvores de Eucalyptus. Sci. For. (Brasil). 42(104):623-629.

23. MURILLO, O.; ESPITIA, M.; CASTILLO, C. 2012. Fuentes semilleras para la producción forestal. Universidad de Córdoba. Ed. Damar S.A.S (Bogotá). 184p.

24. PALENCIA, G.; MERCADO, T.; COMBATT, E. 2006. Estudio agroclimático del departamento de Córdoba, Facultad de Ciencias Agrícolas. Ed. UNICOR (Colombia). 126p.

25. PARAMESH, M.D.M.; REDDY, M.; SHANTHI P.; SUDHAKAR P.; NARASIMHULU, R. 2014. Analysis of genetic parameters for morphophysiological traits in mungbean (Vigna radiata L. Wilczek). Indian Journal of Plant Sciences. 3(2):1-6.

26. RODRIGUES, J.K.; MENDONCA, M.S. DE; GENTIL, D.F. DE O. 2015. Aspectos biométricos, morfoanatômicos e histoquímicos do pirênio de Bactris marajá (Arecaceae). Rodriguésia. (Brasil). 66(1):75-85.

27. SALLA, V.P.; DANNER, M.A.; CITADIN, I.; ZOLET, S.A.S.; DONAZZOLO, J.; GIL, B.V. 2015. Análise de trilha em caracteres de frutos de jabuticabeira. Pesq. Agropec. Bras. 50(3):218-223. 
28. SANTOS, P.E.T. DOS.; FILHO, E.P.; DASILVA, L.T. DE M.; VANDRESEN, P.B. 2015. Genetic variation for growth and selection in adult plants of Eucalyptus badjensis. Genetics and Molecular Biology. (Brasil). 38(4):457-464.

29. SHAFIQUE, M.S.; AHSAN, M.; MEHMOOD, Z.; ABDULLAH, M.; SHAKOOR, A.; AHMAD, M.I. 2016. Genetic variability and interrelationship of various agronomic traits using correlation and path analysis in Chickpea (Cicer arietinum L.). Academia J. Agricultural Research. (USA). 4(2):082-085.

30. SILVA, B.M.; SILVA, C.D.; MORO, F.V.; VIEIRA, R.D. 2015. Morphoanatomy of fruit, seed and seedling of Ormosia paraensis Ducke. J. Seed Sci. (Brasil). 37(4):192-198.

31. SILVA, K.B.; ALVES, E.U.; MATOS, V.P.; BRUNO, R.L. 2012. Caracterização morfológica de frutos, sementes e fases da germinação de Pachira aquatica Aubl, (Bombacaceae). Rev. Ciênc, Agr. (Brasil), 33(3):891898.

32. SILVA, M. DE S.; BORGES, E.E. DE L.; LEITE, H.G.; CORTE, V.B. 2013. Biometria de frutos e sementes de Melanoxylon brauna Schott. (FabaceaeCaesalpinioideae). CERNE. (Brasil). 19(3):517-524.
33. SOLANGI, S.K.; QURESHI, S.T.; SOLANGI, N.; SOLANGI, M.K.; KHAN, I.A.; PARVEEN, A.I.; PARVEEN, A.CH.; SOLANGI, A.H. 2016. Heritability analysis to screen elite sugarcane (Saccharum spp.) soma clones under field condition. J. Plant Breeding and Crop Science. (USA). 8(9):168-174.

34. SOUZA, D.K.; LIMA, R.A.; DOMINGUES, C.A.; PEDROSO, L.A.; FACUNDO, V.A.; GAMA, F.C.; ALVES, M.R. 2014. Potencial fungicida do extrato etanólico obtido das sementes de Pachira aquatica Aubl. sobre Fusarium sp. Ciência e Natura. (Brasil). 36(2):114-119.

35. VALE, I. DO; COSTA, L.G.S.; MIRANDA, I.S. 2014. Espécies indicadas para a recomposição da floresta ciliar da sub-bacia do rio peixe-boi, Pará. Ciência Florestal. (Brasil). 24(3):573-582.

Recibido: Diciembre 18 de 2017

Aceptado: Mayo 1 de 2018

\section{Cómo citar:}

Espitia-Camacho, M.; Araméndiz-Tatis, H.; Cardona-Ayala, C. 2018. Parámetros genéticos de las caracteristicas biometricas del fruto y semillas en Pachira aquatica Aubl. Rev. U.D.C.A Act. \& Div. Cient. 21(1): 33-42. 